\title{
A PHYSIOLOGIC JOURNEY
}

\section{Cheng HM}

Department of Physiology, Faculty of Medicine, University of Malaya, Kuala Lumpur

\section{Correspondence:}

Professor Dr. Cheng Hwee Ming

Department of Physiology

Faculty of Medicine, University of Malaya, 50603 Kuala Lumpur.

E-mail: chenghm@um.edu.my

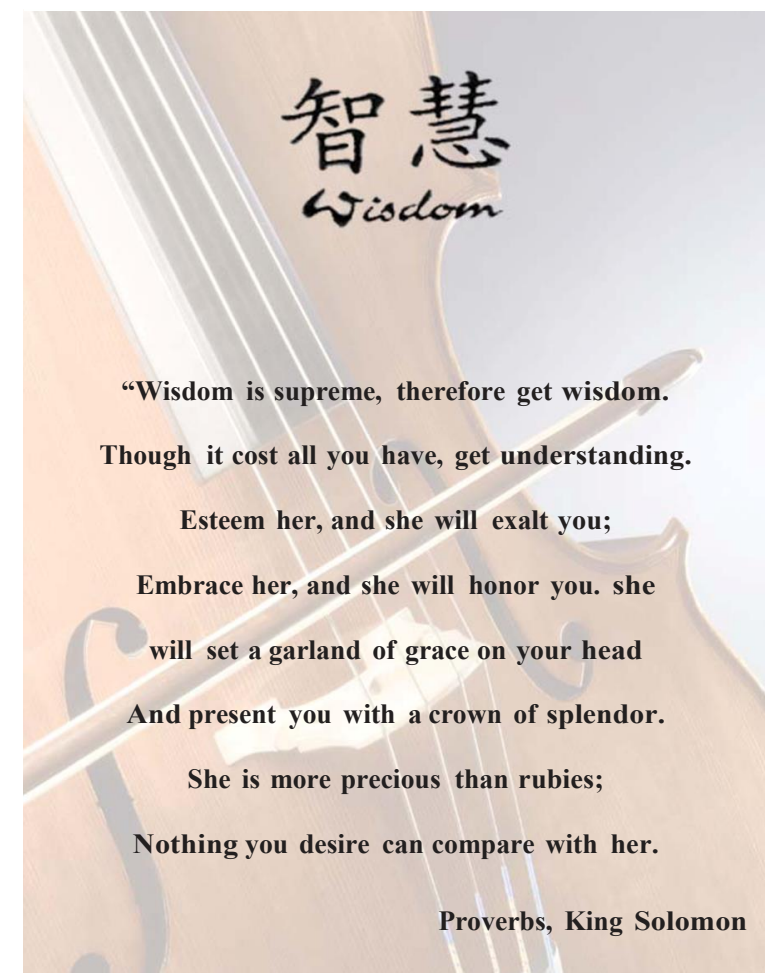

\section{A Physiologic Journey}

".... You knit me together in my mother's womb".

The Psalmist.

My first systematic introduction to laboratory research began with my post-graduate studies at the Department of Immunology, Royal Liverpool Hospital in 1980. My PhD mentor was Professor Peter Johnson, an inspiring teacher and I learnt much from his approach to addressing research questions and his ability to give meaning to seemingly disjointed data. Peter, as I called him has since retired and happily judges sailing competitions.Above Peter's desk were the words of immunologist, JR Marrack, "The secret of successful research is to ask nature, simple questions, one at a time". I was introduced to the mysteries surrounding the natural phenomenon of placental implantation. The placenta, being a 'foreign' fetal allograft, not merely survives passively against any potential maternal immune rejection, but actively provides the nutritive, hormonal and homeostatic lifeline for a relatively long gestation in humans. The placenta is aptly described as 'Nature's Transplant' and research insights into the immunobiology of the successful placental allograft may provide better understanding of how cancer cells avoid host immunosurveillance. I remember my regular visits to the Women's Hospital, Liverpool to collect fresh, still warm samples. The use of a large, easily available human sample has a great advantage as it removes doubts when the relevance of extrapolating animal data to human is concerned. There are obvious expectations that there will be a cross-pollination of knowledge from placental research to human organ transplantation. My PhD involved using monoclonal antibodies produced against the placental syncytiotrophoblast membrane. 


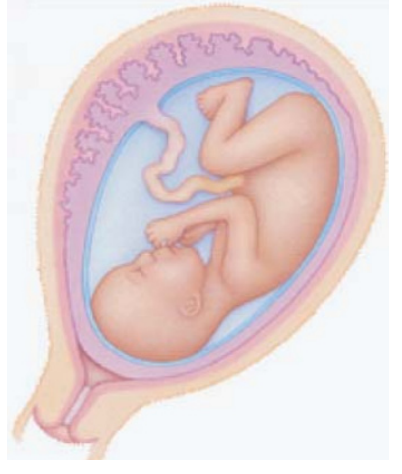

I was proud to publish my first author paper in the journal Placenta (Cheng HM, Johnson PM. 1985).

In 1985, on returning home to Malaysia, I joined the Physiology Department then chaired by the late Professor A Raman. There was not a Division of Immunology in the Faculty of Medicine for me to consider applying for. As is the case for many PhDs in the Life Sciences who returned in the early 80's to Malaysian public universities, lack of appropriate research facilities and funds did not permit the continuation of my investigation into the placenta (although of course, the supply of human placentae would not have been a problem!). I subsequently met Prof Yap Sook Fan who was at that time attempting to set up a radio-immunoassay for antibodies to the mitochondrial phospholipid, cardiolipin. The anticardiolipin syndrome was beginning to be recognized as a definite autoimmune entity.

"Any kingdom divided against itself will be ruined ....". Luke the Physician

In the process of testing for anticardiolipin antibodies $(\mathrm{aCL})$ in clinical samples and establishing an enzymelinked immunoassay (ELISA) for aCL, we serendipitiously observed aCL in normal human sera. This providential finding led to characterization of these physiologic antiphospholipid autoantibodies. The obvious question was the immunological relevance of these natural antibodies in health and disease. At that time, there was progressively accumulating descriptions of natural antibodies to other normal tissue components in the literature. In addition, the natural antibodies are in some cases masked, latent or cryptic and are detectable only when serum components that appear to bind them are removed. Interestingly, the antigenic specificities of these natural

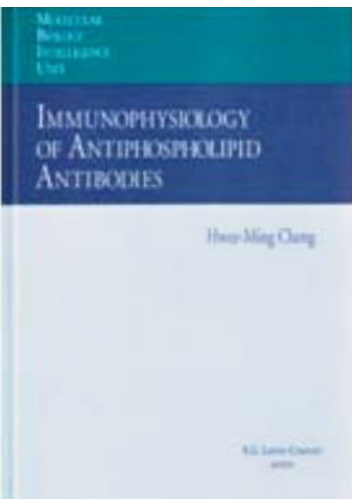
antibodies (including $\mathrm{aCL}$ ) were similar to those found in autoimmune sera. One paradigm shift views the subpopulation of circulating natural antibodies as an essential part of the i m munoregulatory network operative in health. In other words, when a specific antibody is detected in e.g. an SLE patient, the presence of that antibody may not necessarily be due to solely a hyperimmune response to antigen. The autoimmune autoantibody could also be exposed and detected when the serum masking factors are reduced, and the cryptic natural antibody with the same specificity then identified by laboratory immunoassays. These studies were satisfying and productive and an invited authorship by a specialty book publisher followed with the release in 1994 of "Cheng HM, Immunophysiology of Antiphospholipid Antibodies".

During the course of antiphospholipid investigations, there were some forays into humoral immunity in cancer and in allergy. My friend and scientific colleague, Prof Sam Choon Kook kindly absorbed me into her laboratory team, then at the ENT department under Prof U Prasad. Antibodies to antigens of the Epstein Barr virus (EBV) were of special interest in early screening of the EBV-related nasopharyngeal carcinoma (NPC). Efforts were also made into elucidating the fine linear antigenic epitopes of EBV antigens recognized by antibodies in NPC patients. The aim of such immunomapping was to utilize simplified, immunodominant linear peptides in immunoassay for NPC screening.

\section{$1997-2010$}

"Fruit trees of all kinds.... will serve for food and their leaves for healing"

Ezekiel the Prophet

In 1997, I spent my Sabbatical at the then Palm Oil Research Institute (now Malaysian Palm Oil Research Board). Dr Kalyana Sundram initiated me into Nutrition research and in particular, the research focus was on palm oil-derived vitamin E. Vitamin E besides its antioxidant properties has other biological actions. Oxidation studies of low-density lipoprotein were carried out and the possible roles of natural antibodies in immunoclearance of oxidized LDL were proposed. Carefully controlled human volunteer projects, including post-prandial studies with different palm vitamin E fractions were also conducted.

Together with colleagues in SIRIM and International Medical University, we also optimized several tests to screen for net free radical scavenging activities in Malaysian natural products. One surprising finding that was eventually patented was the detection of a high level of antioxidant activity in the uneaten skins of our common rambutan fruit.

An important question relates to the bioavailability of dietary antioxidants. Some investigations in intestinal absorption in rats were carried out using extracts of the Mulberry plant as the antioxidant source. We encountered serendipitiously what might be an important natural antioxidant defence phenomenon in isolated intestinal preparation (unpublished). 
In 2003, while enjoying my $3^{\text {rd }}$ Sabbatical, I initiated the $1^{\text {st }}$ Inter-Medical School Physiology Quiz (IMSPQ). From

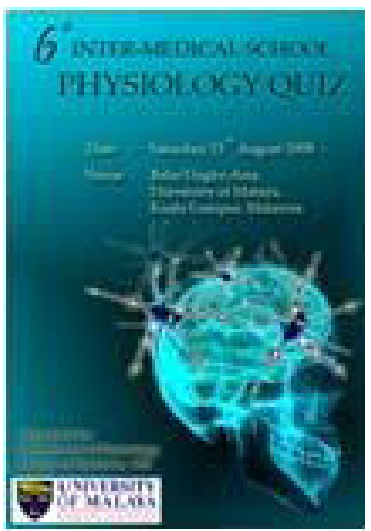
an initial small competition of only 7 national medical schools, the IMSPQ has bloomed and expanded. In the recent $20108^{\text {th }}$ IMSPQ, we had 40 medical university teams from 15 countries participating. The IMSPQ is now an annual global gathering of both medical students and lecturers hosted proudly by the Department of Physiology, Universiti Malaya. As Organizing Chairman of the IMSPQ, it has been most fulfilling to be part of the unique gathering of medical students and Physiological educators. The American Physiological Society has recognized this stimulus for encouraging Physiology learning and has invited a write-up of the event in their "Advances in Physiology Education" journal (Cheng HM, 2010).

"The Teacher searched to find just the right words,
...."

Ecclesiastes

After 15 years of teaching and reading Physiology, it was time to summarize and concretize some of the insights gained from seeing how students learn. It is well said that 'Writing focuses the mind'. In 2000 I began writing and formulating questions in diverse ways to help students assimilate major Physiological concepts as well as to highlight common misconceptions in integrating Physiological mechanisms in a dynamic subject. Students truly are our best teachers. Tutorial and walk-in questions and answers verbalized by the students provide a window into how they are processing the myriads of information. Even ridiculous answers in examination papers can sometime provide such a gem if one ponders a little. The time spent with

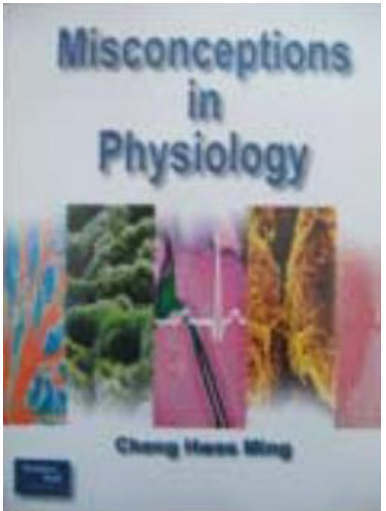
and the effort put into listening to students have been enriching. The fruits of this joyful labour are part of the 16 Physiology learning books that have been written.

In describing my 'Physiologic Journey', I decided to list some major milestones along the way. Milestones are often seen as achievements and they are worthy of some praise. Among the milestones on my journey, there are some that are happily beyond my control. Especially are the wonderful gifts of my two children Timothy and Ruth and my friend and companion for life, Gaik Cheng.

The journey continues in the land of Academia...

"All their life in Narnia had been the cover and the title page: now at last they were beginning Chapter One of the Great Story which no one on earth has read: which goes on forever: in which every chapter is better than the one before".

The Last Battle, CS Lewis

The text was presented in an inaugural lecture by Professor Dr. Cheng Hwee Ming in the Faculty of Medicine, University of Malaya on the 1st April 2011. 